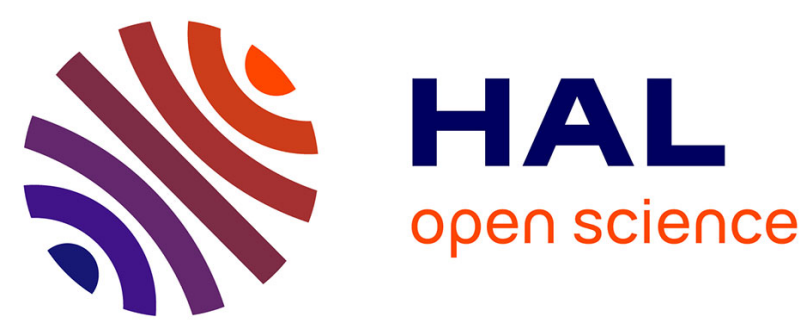

\title{
Reduced-order representation of near-wall structures in the late transitional boundary layer
}

\author{
Taraneh Sayadi, Peter J. Schmid, Joseph W. Nichols, Parviz Moin
}

\section{To cite this version:}

Taraneh Sayadi, Peter J. Schmid, Joseph W. Nichols, Parviz Moin. Reduced-order representation of near-wall structures in the late transitional boundary layer. Journal of Fluid Mechanics, 2014, 748 (june), pp.278-301. 10.1017/jfm.2014.184 . hal-01050701

\section{HAL Id: hal-01050701}

https://hal-polytechnique.archives-ouvertes.fr/hal-01050701

Submitted on 25 Jul 2014

HAL is a multi-disciplinary open access archive for the deposit and dissemination of scientific research documents, whether they are published or not. The documents may come from teaching and research institutions in France or abroad, or from public or private research centers.
L'archive ouverte pluridisciplinaire HAL, est destinée au dépôt et à la diffusion de documents scientifiques de niveau recherche, publiés ou non, émanant des établissements d'enseignement et de recherche français ou étrangers, des laboratoires publics ou privés. 


\title{
Reduced-order representation of near-wall structures in the late transitional boundary layer
}

\author{
Taraneh Sayadi ${ }^{1,}$, Peter J. Schmid ${ }^{2}$, Joseph W. Nichols ${ }^{3}$ and Parviz Moin ${ }^{4}$ \\ ${ }^{1}$ Laboratoire d'Hydrodynamique (LadHyX), Ecole Polytechnique, 91128 Palaiseau, France \\ ${ }^{2}$ Department of Mathematics, Imperial College London, South Kensington Campus, \\ London SW7 2AZ, UK \\ ${ }^{3}$ Department of Aerospace Engineering and Mechanics, University of Minnesota, \\ Minneapolis, MN 55455, USA \\ ${ }^{4}$ Centre for Turbulence Research (CTR), Stanford University, Stanford, CA 94305, USA
}

(Received 3 July 2013; revised 25 March 2014; accepted 1 April 2014; first published online 28 April 2014)

\begin{abstract}
Direct numerical simulations (DNS) of controlled $\mathrm{H}$ - and K-type transitions to turbulence in an $M=0.2$ (where $M$ is the Mach number) nominally zero-pressuregradient and spatially developing flat-plate boundary layer are considered. Sayadi, Hamman \& Moin (J. Fluid Mech., vol. 724, 2013, pp. 480-509) showed that with the start of the transition process, the skin-friction profiles of these controlled transitions diverge abruptly from the laminar value and overshoot the turbulent estimation. The objective of this work is to identify the structures of dynamical importance throughout the transitional region. Dynamic mode decomposition (DMD) (Schmid, J. Fluid Mech., vol. 656, 2010, pp. 5-28) as an optimal phase-averaging process, together with triple decomposition (Reynolds \& Hussain, J. Fluid Mech., vol. 54 (02), 1972, pp. 263-288), is employed to assess the contribution of each coherent structure to the total Reynolds shear stress. This analysis shows that low-frequency modes, corresponding to the legs of hairpin vortices, contribute most to the total Reynolds shear stress. The use of composite DMD of the vortical structures together with the skin-friction coefficient allows the assessment of the coupling between near-wall structures captured by the low-frequency modes and their contribution to the total skin-friction coefficient. We are able to show that the low-frequency modes provide an accurate estimate of the skin-friction coefficient through the transition process. This is of interest since large-eddy simulation (LES) of the same configuration fails to provide a good prediction of the rise to this overshoot. The reduced-order representation of the flow is used to compare the LES and the DNS results within this region. Application of this methodology to the LES of the H-type transition illustrates the effect of the grid resolution and the subgrid-scale model on the estimated shear stress of these low-frequency modes. The analysis shows that although the shapes and frequencies of the low-frequency modes are independent of the resolution, the amplitudes are underpredicted in the LES, resulting in underprediction of the Reynolds shear stress.
\end{abstract}

Key words: boundary layer structure, transition to turbulence

$†$ Email address for correspondence: tsayadi@imperial.ac.uk 


\section{Introduction}

Traditionally, turbulence models rely on statistical or averaged quantities. Although valuable for many applications, these models commonly ignore the presence of coherent structures in the flow despite the fact that these structures are known to be pivotal to our understanding of the flow dynamics and are responsible for maintaining turbulence through production and dissipation mechanisms. The dynamics of coherent structures should play an important role in the design of more predictive models for turbulent flow, in addition to establishing a link between organized fluid motion and turbulence statistics.

Coherent structures in turbulent boundary layers were first uncovered and quantitatively described in the early 1930s through experimental observations. Since then, many experimental (Kline et al. 1967; Head \& Bandyopadhyay 1981; Borodulin, Kachanov \& Roschektayev 2011) and numerical (Rist \& Fasel 1995; Wu \& Moin 2009; Sayadi, Hamman \& Moin 2013) studies of transitional and turbulent boundary layers have reaffirmed that indeed coherent structures are present in these flows and play an important role in the mass, momentum and energy transport (for a review we refer to Hussain (1986) and Robinson (1991)). There has been a great deal of effort to extract coherent structures from experimental and numerical data; however, many of the identification methods rely on a priori knowledge of the specific form of the structure. One method for the extraction of spatial modes while still avoiding subjectivity is proper orthogonal decomposition (POD) (Bakewell \& Lumley 1967; Sirovich 1987; Holmes, Lumley \& Berkooz 1996). Since it is based on a sequence of flow snapshots, POD is equally applicable to experiments and numerical simulations. Through this procedure the most energetic modes are extracted which then form a hierarchical basis to describe the flow. In a subsequent step, the POD basis can be truncated to obtain low-dimensional systems of ordinary differential equations from the Navier-Stokes equations via Galerkin projections. This approach has been used by Aubry et al. (1988) to represent the dynamics of near-wall structures in a turbulent boundary layer. Using similar techniques, Moin \& Moser (1989) extracted three-dimensional coherent structures from direct numerical simulations (DNS) of channel flow via POD. Despite its early concentration on turbulent fluid motion, POD has also been applied to transitional flows, see e.g. Rempfer \& Fasel (1994). The majority of POD studies, however, have focused on identifying structures in near-wall turbulence or on detecting three-dimensional structures arising from a secondary instability in transitional flows. In this article, we are interested in identifying the structures responsible for the rise of the transitional skin-friction profile of a boundary layer, in tracing them through the peak in the skin friction and in quantifying their individual contributions to the total Reynolds shear stress.

A ranking of fluid structures by energy, followed by a truncation that retains only the most energetic components, cannot account for structures that are low energy but nonetheless contribute significantly to the flow dynamics. In this respect, dynamic mode decomposition (DMD) (Schmid 2010) constitutes a suitable alternative to POD as it extracts spatial modes based on their single-frequency content rather than sorting them according to their contribution to the total energy. Dynamic mode decomposition has been applied in the context of turbulent channel flow to extract coherent structures from the flow by Mizuno et al. (2011); however, the broadband nature of the spectra in that scenario makes it ill-suited for DMD. The first objective of the present work is to show that DMD is capable of extracting relevant coherent structures from transitional flows, in particular from a DNS of H- and K-type controlled transitions of a compressible boundary layer (Sayadi et al. 2013). Both H- and K-type transitions are 


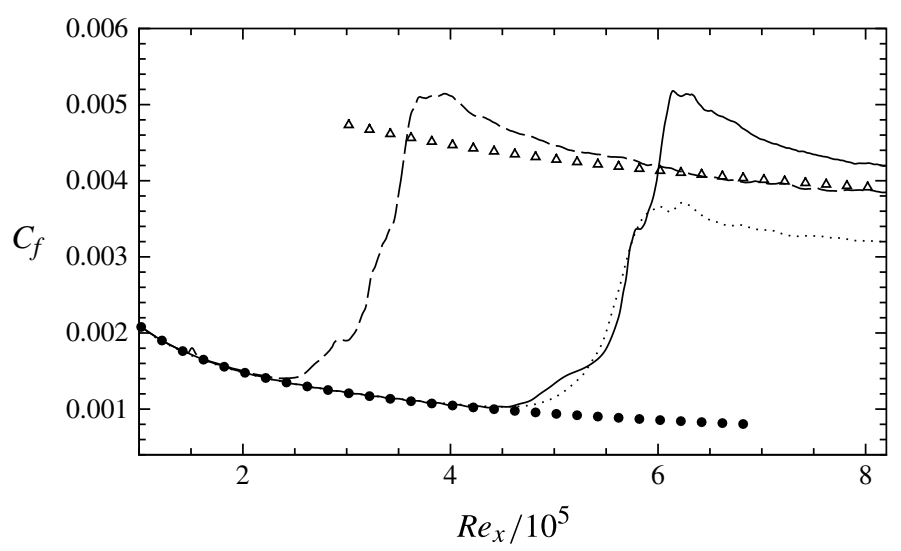

FIgURE 1. The skin-friction coefficient versus $R e_{x}$ : --, H-type transition; ---, K-type transition; - laminar correlation; $\Delta$, turbulent correlation (White 2006); $\cdots$, large-eddy simulation (LES) H-type transition.

examples of transitions in which prescribed low-amplitude waves, confined near the wall, develop without external perturbations. Experiments by Schubauer \& Skramstad (1947), Klebanoff, Tidstrom \& Sargent (1962) and Kachanov \& Levchenko (1984) demonstrated that the interaction of two-dimensional Tollmien-Schlichting (TS) waves with lower-amplitude oblique waves results in three-dimensionality in the flow. This interaction is well described by the linear Orr-Sommerfeld equation and precedes the development of $\Lambda$-vortices (Berlin, Wiegel \& Henningson 1999; Bake, Fernholz $\&$ Kachanov 2000). Here, $\Lambda$-vortices have two elongated legs of counter-rotating streamwise vorticity and a tip of spanwise vorticity that is characteristic of hairpin vortices. In contrast to the K-type transition, where these vortices are aligned, the $\mathrm{H}$-type transition is characterized by staggered arrangements of $\Lambda$-vortices (Saric 1986). The structures that appear in the early stages of these transitional regimes are well documented, both experimentally and numerically (Borodulin \& Kachanov 1995; Rist \& Fasel 1995), which makes them suitable for validation purposes.

Moreover, DMD can be considered as an optimal phase-averaging process and can be used in the context of a triple decomposition (Reynolds \& Hussain 1972), further described in $\S 4.1$, to directly account for the contribution of the coherent structures to the nonlinear term in the Navier-Stokes equations. This is of particular relevance as coherent structures can be extracted from wall-bounded flows using different techniques; however, any such effort would be of limited use unless it can be shown that these extracted structures contribute notably to the dynamics of the underlying turbulent flow. Therefore, the application of DMD to the late transitional regime, which is marked by a significant rise in the skin-friction coefficient, as shown in figure 1, would help in identifying the coherent structures associated with this rise and provide a reduced-order representation of the flow structures. Preliminary studies of the two-dimensional skin-friction data through the late transitional regime, using DMD (Sayadi et al. 2012), showed that the plateau that appears at $R e_{x} \approx 5.7 \times 10^{5}$, where $R e_{x}$ is the Reynolds number based on the distance from the leading edge of the plate, coincides with the first appearance of hairpin-like structures, and that the trace of these structures is successfully reproduced using a few low-frequency DMD modes. This motivated us to pursue DMD of the full three-dimensional velocity field. Additionally, the dynamical significance of these structures is directly assessed by 
evaluating their contribution to the total Reynolds shear-stress gradient. A noteworthy advantage of the DMD algorithm is its flexibility in processing a time-synchronous composite set of data, consisting, e.g., of the $Q$-criterion, representing the vortical structures, and the skin friction at the wall. Accordingly, our aim is to establish a direct link between vortical flow structures and their trace on the skin-friction coefficient, and, as a result, to quantify the coupling between the dynamically important structures and the skin-friction coefficient.

The skin-friction profiles of the $\mathrm{H}$ - and K-type transitions, as shown in figure 1 , exhibit a distinct overshoot of the turbulent drag coefficient in the late transition region. This figure also shows that after reaching the overshoot, the skin-friction value converges gradually towards the predicted value for the turbulent boundary layer. This behaviour is most obvious in the skin-friction profile taken from the K-type transition. Recent studies by Sayadi \& Moin (2012) showed that dynamic subgrid-scale (SGS) models accurately capture the onset of transition in controlled $\mathrm{H}$ - and K-type transition scenarios. However, as illustrated in figure 1, coarse LES calculations underpredict both the overshoot in the skin-friction coefficient during the late stages of transition and the skin-friction equilibrium level during the ensuing turbulent regime. Dynamic mode decomposition is applied to under-resolved Navier-Stokes (UNS) and LES calculations of the H-type transition, keeping the configuration similar to that of the DNS. Comparison of the resulting shear stress from the low-frequency modes of the UNS and LES with that of the DNS provides insight into the effect of the lower resolution and the model on the structures of the stress-producing modes. During the late stages of transition, deterministic structures are responsible for producing the bulk of the turbulent flow statistics (see Sayadi et al. 2013), lending support to a link via coherent structures between the late transitional regime and fully developed fluid motion.

This paper is organized as follows. In $\S 2$ the computational set-up used in the DNS calculations is briefly described, including a list of all grids used in the calculations. In $\S 3$ DMD is applied to DNS snapshots of the early transitional flow, illustrating the extracted dominant structures and their ability to capture the principal dynamical processes in a low-dimensional manner. Section 4 focuses on a DMD analysis from a triple-decomposition point of view, where DNS data describing the rise of the skin-friction coefficient will be processed. We will see that, with only a few low-frequency modes, the near-wall structures of the transitional boundary layer can be accurately captured. By using a composite DMD, the footprint of these near-wall vortical structures on the skin-friction coefficient can be identified. The effect of diminished resolution and the use of SGS models on these low-frequency modes is investigated by applying the same methodology to the results of low-resolution LES and UNS in $\S 5$. Finally, in $\S 6$ conclusions from the current study are given.

\section{Computational set-up}

A sketch of the computational domain is shown in figure 2 . The fully compressible Navier-Stokes equations are solved for a perfect gas using fourth-order finite differences with Mach number $M=0.2$ (Sayadi 2012). Although the fully compressible Navier-Stokes equations were used to simulate the boundary layer, the relatively low free-stream Mach number implies that compressibility effects are not significant. Indeed, we have confirmed that compressibility effects are negligible by examining the growth rate of the low-amplitude perturbations in the linear and weakly nonlinear regimes. In a recently published work involving the same dataset (Sayadi et al. 2013), 


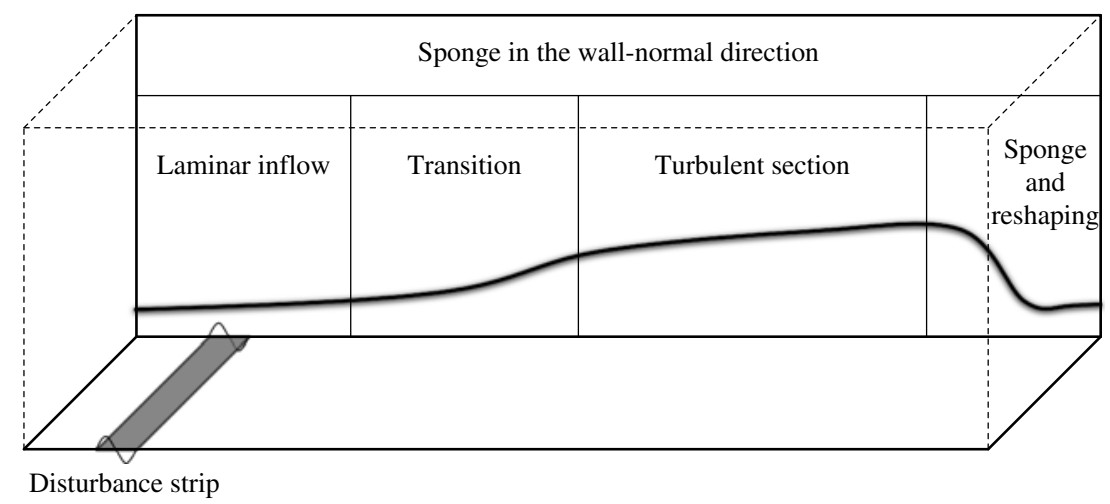

FIGURE 2. Sketch of the computational domain.

$\begin{array}{lcccccccccc} & L_{x} & L_{y} & L_{z} & N_{x} & N_{y} & N_{z} & \Delta x^{+} & \Delta y_{\min }^{+} & \Delta z^{+} & M \\ \text { H-type DNS } & 9.6 & 1.0 & 0.60 & 4096 & 550 & 512 & 10 & 0.4 & 5.4 & 0.2 \\ \text { K-type DNS } & 8.6 & 0.9 & 0.60 & 4096 & 550 & 512 & 10 & 0.4 & 5.5 & 0.2 \\ \text { H-type LES/UNS } & 9.6 & 0.5 & 0.15 & 480 & 160 & 16 & 90 & 0.9 & 42 & 0.2\end{array}$

TABLE 1. Characteristics and numerical parameters of the $\mathrm{H}$ - and K-type transitions. The friction velocity, $u_{\tau}$, used for the calculation of the wall units is taken at the location where the skin friction converges to the value predicted by the turbulent correlation (Sayadi \& Moin 2012).

we found that the evolution of the perturbations in the early transitional regime agrees with the evolution of the perturbations in $\mathrm{H}$ - and K-type experiments (Kachanov \& Levchenko 1984). In addition, the turbulence statistics in the fully turbulent section of the present simulation compare well with equivalent statistics taken from the incompressible DNS of $\mathrm{Wu} \&$ Moin (2009). The hybrid implicit-explicit time integration scheme employs a third-order Runge-Kutta (RK3) scheme for the explicit time advancement and a second-order A-stable scheme for the implicit time-stepping. The numerical scheme is designed for a structured curvilinear grid and the variables are staggered in space (Nagarajan, Lele \& Ferziger 2003). The inlet Reynolds number based on the distance from the leading edge is $R e_{x}=10^{5}$ and the distance of the inlet station from the leading edge of the plate, $x_{0}$, is used as the characteristic length scale, i.e. $x / x_{0}=R e_{x} / 10^{5}$. Details of the computational domains are given in table 1 , where $L$ and $N$ are the length and the number of grid-points along each spatial direction, respectively. The grid resolutions are also reported in plus unit, denoted by $\Delta x^{+}, \Delta y_{\min }^{+}$and $\Delta z^{+}$.

Periodic boundary conditions are used in the spanwise and streamwise directions. To allow for a spatially growing boundary layer in a streamwise-periodic domain, the profile at the end of the domain is reshaped and rescaled into the similarity solution of a laminar compressible boundary layer at the inflow. This reshaping procedure is performed inside a sponge region, following the earlier work by Spalart \& Watmuff (1993). Inside all sponge zones, which are situated at the top, inlet and outlet of the computational domain, the similarity solution of the compressible laminar boundary layer is prescribed. For more details on the direct and large-eddy simulations see Sayadi \& Moin (2012) and Sayadi et al. (2013). 


\section{Decomposition methodology}

In this section, we describe the transitional boundary layer by a truncated modal decomposition of a time sequence of flow fields. This decomposition divides the spatio-temporal process into a basis of coherent spatial modes and provides amplitude information as well as a temporal signal associated with each spatial mode. The nature of the decomposition ensures that each individual mode satisfies the proper boundary conditions, and the entire snapshot sequence can be reconstructed by a superposition of the individual components. Since the amplitude information from the decomposition is expected to identify more dominant spatial modes, a representation with only the most prevalent structures (and their temporal dynamics) will yield a low-dimensional reduced-order model of the decomposed process. In what follows, we give a brief description of the chosen DMD formalism, after which we apply it to DNS of the early stages of $\mathrm{H}$ - and K-type transitions where documented flow structures exist.

\subsection{Dynamic mode decomposition: formalism}

Many fluid processes show a great deal of complexity, yet are governed by the dynamics of a few coherent flow states. The role of model reduction is the detection of these states and the approximate description of the full process by a substantially reduced number of degrees of freedom, which still captures the 'essential physics' of the original fluid system.

As an example, if $U(x, y, z, t)$ is a flow variable, where $x, y$ and $z$ are the spatial coordinates and $t$ is time, we can generally decompose the flow field into

$$
U(x, y, z, t)=\sum_{n=1}^{N} a_{n} \phi_{n}(x, y, z) \psi_{n}(t),
$$

where $\phi_{n}$ denotes the $n$th spatial mode (normalized to unit norm), $a_{n}$ represents the amplitudes and $\psi_{n}$ stands for the temporal behaviour of the respective modes. This general decomposition is not unique, and further assumptions on the spatial modes or their temporal behaviour have to be made.

The choice of requiring $\psi_{n}(t)$ to consist of only a single (complex) frequency, $\lambda=\lambda_{r}+\mathrm{i} \lambda_{i}$, yields the DMD. This technique is the basis of Koopman analysis of nonlinear dynamical systems (Mezic 2005; Rowley et al. 2009). The Koopman operator is a linear operator that can be defined for any nonlinear system and is not based on linearization. For time-periodic data it is equivalent to a Fourier transform in time. Dynamic mode decomposition only requires a sequence of flow snapshots and can thus be applied to data sequences from experiments and simulations alike. For data sequences from a linearized flow simulation, it is equivalent to a global stability analysis; for data sequences from a nonlinear flow, it produces modes of the tangential linear approximation of the full system matrix and describes dominant dynamic behaviour contained in the data sequence.

Dynamic mode decomposition extracts a low-dimensional description of a linear transformation that maps any snapshot from the data sequence into the subsequent snapshot. The eigenvalue decomposition of this linear transformation gives frequency information (from the eigenvalues, via a logarithmic mapping) as well as spatial structures (from the eigenvectors). A projection of the data sequence onto these structures provides amplitude information, i.e. information on how much each identified structure is represented in the original data sequence. More details can be found in Rowley et al. (2009), Schmid (2010) and Schmid, Violato \& Scarano (2012). 

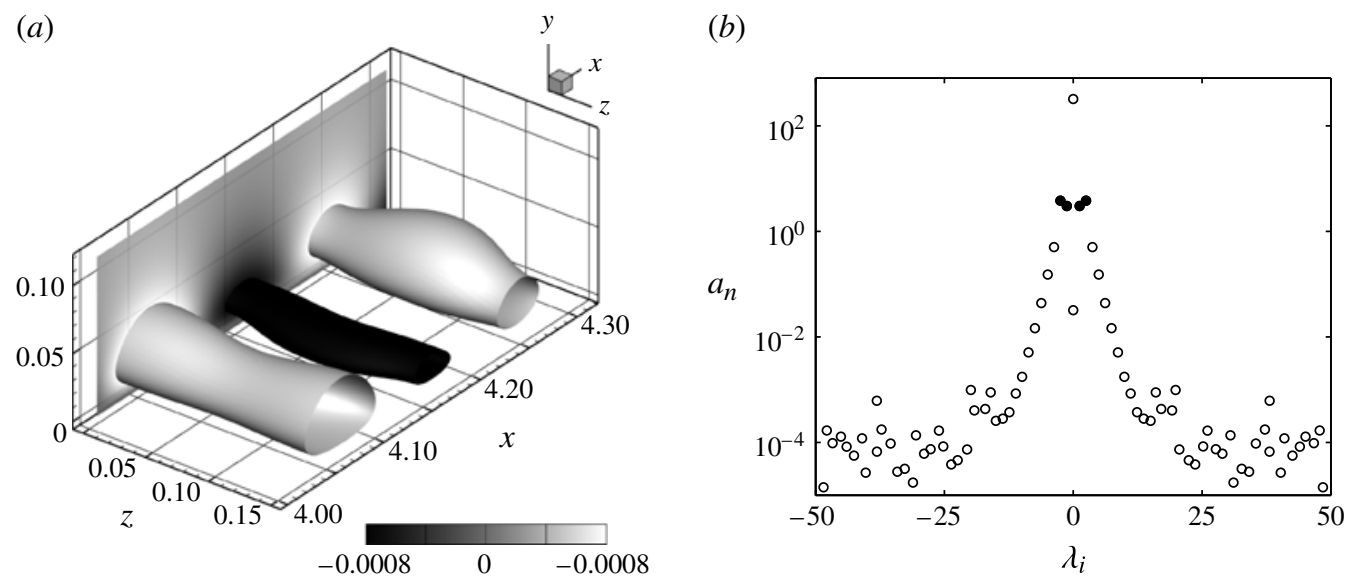

FIGURE 3. The iso-structures of the wall-normal velocity $(a)$ and the corresponding DMD spectrum $(b)$ for all modes (empty circles) and the TS and corresponding subharmonic waves (filled circles) from an analysis of the three-dimensional domain extending from $x / x_{0}=4.0$ to $x / x_{0}=4.3$ (H-type).

\subsection{Application to early transitional flow}

Two points need to be addressed in the context of a spatially growing transitional boundary layer. First, due to the convective instability of the flow, modes grow or decay downstream, and in order to represent the coherent structures in the flow accurately, DMD must capture this behaviour. Furthermore, we have mentioned that each DMD mode is a representation of a spatial structure with a specific single frequency. In the special case of controlled transition, fundamental and subharmonic modes of known frequency are introduced into the flow through blowing and suction; for this reason, a first validation case would be to check whether DMD is capable of identifying the shape and frequencies of these modes accurately. These two points are addressed here by examining the early stages of H-type transition.

Dynamic mode decomposition is performed on the three velocity components of the DNS data. The computational domain for the DNS is divided into 32 equidistant subdomains in the streamwise direction with $\Delta x / x_{0}=0.3$. This streamwise length covers at least one wavelength of the TS wave $\left(\Delta_{T S}=0.2\right)$. In the laminar and early transition section, only one spanwise wavelength of the subharmonic wave is included in the domain under consideration, such that $\Delta z=L_{z} / 4$. The wall-normal range of the DMD subdomain extends to the edge of the boundary layer. One hundred and one temporally equispaced snapshots spanning two periods of the fundamental frequency are extracted from the respective simulations.

Figure 3(a) covers the subdomain extending from $x / x_{0}=4.0$ to $x / x_{0}=4.3$. The skin-friction profile for this streamwise range coincides with the laminar solution, even though nonlinear interactions leading to the growth of the subharmonic waves have already taken place, causing a three-dimensional modulation of the flow. The isosurfaces of the wall-normal velocity, depicted in figure $3(a)$, confirm this fact. The DMD spectrum, displaying the amplitude of each mode versus the imaginary frequency, is plotted in figure $3(b)$. Since only real data have been processed, the identified frequencies come in complex-conjugate pairs; this is also reflected in 
(a)

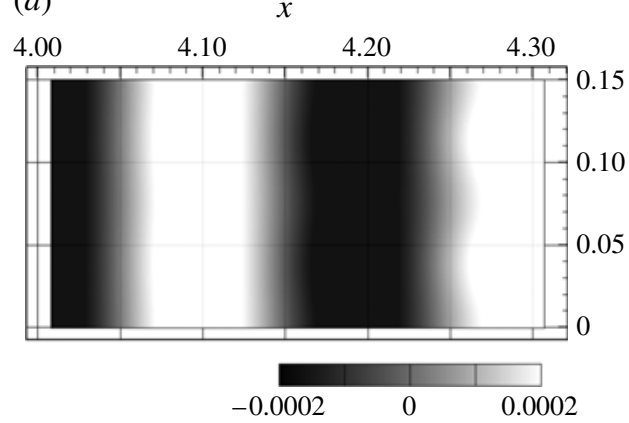

(b)

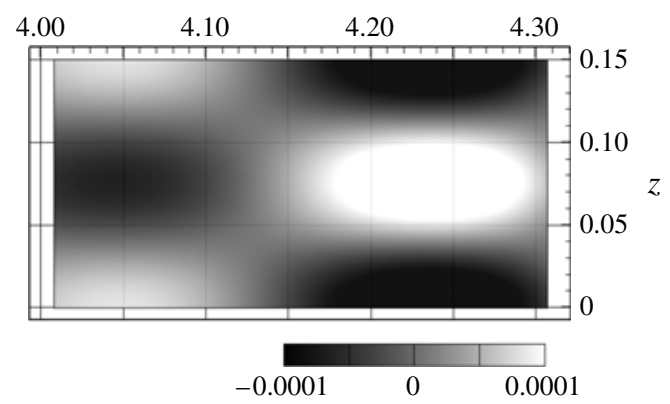

FIGURE 4. Contours of the wall-normal velocity at $y / x_{0}=0.01$ (H-type) for the two dominant dynamic modes (indicated by filled symbols in figure $3(b)$ ), extracted from the domain extending from $x / x_{0}=4.0$ to $x / x_{0}=4.3$ : (a) 2-D TS wave, $(b)$ subharmonic wave.

$\begin{array}{lcc} & \lambda_{T S} & \lambda_{S H} \\ \text { Blowing and suction strip } & 1.24 \times 10^{-4} & 0.64 \times 10^{-4} \\ \text { DMD } & 1.24 \times 10^{-4} & 0.64053 \times 10^{-4}\end{array}$

TABLE 2. The frequencies of the fundamental TS and subharmonic waves (H-type transition), introduced in the DNS via a blowing/suction strip and identified by DMD from a snapshot sequence.

the amplitude distribution in figure $3(b)$. The two eigenfunctions corresponding to the same amplitude and the same absolute frequency represent the same spatial structure: they can be considered as a pair of modes with a phase difference such that in combination the correct advection speed is captured. In the context of this paper they will be considered as a single-mode or coherent structure of the flow. The frequencies of dynamic modes are determined as $\lambda=\omega \nu / U_{\infty}^{2}$, normalized by the molecular viscosity and freestream velocity. The frequencies of the modes corresponding to the largest amplitude (after the mean), shown in figure $3(b)$ with filled circles, are compared to the fundamental TS and subharmonic frequencies in table 2. The results confirm that the DMD is capable of isolating the frequencies of disturbances introduced into the domain. Figure 4 shows the corresponding spatial shapes of these two modes. The first mode, figure $4(b)$, reflects the spatial shape of the subharmonic disturbance, while the second mode captures the TS wave.

The respective amplitudes of the fundamental TS and the subharmonic wave versus the Reynolds number $R e_{x}$ are plotted for comparison in figure 5. They are computed by sampling the data over two temporal periods of the fundamental wave. The sampled data are then Fourier transformed in time and the spanwise direction. The respective amplitudes of the fundamental TS and the subharmonic wave are compared with the predictions of the two DMD modes. The exponential growth of the subharmonic wave is correctly captured by the respective DMD mode. In this region, nonlinear interactions of the disturbances lead to saturation of the TS wave amplitude. The respective DMD mode shows similar behaviour. As a result, we can conclude that DMD captures both the growth and the saturation of perturbations in the early transitional regime. In this way, DMD spans the limiting regimes of 


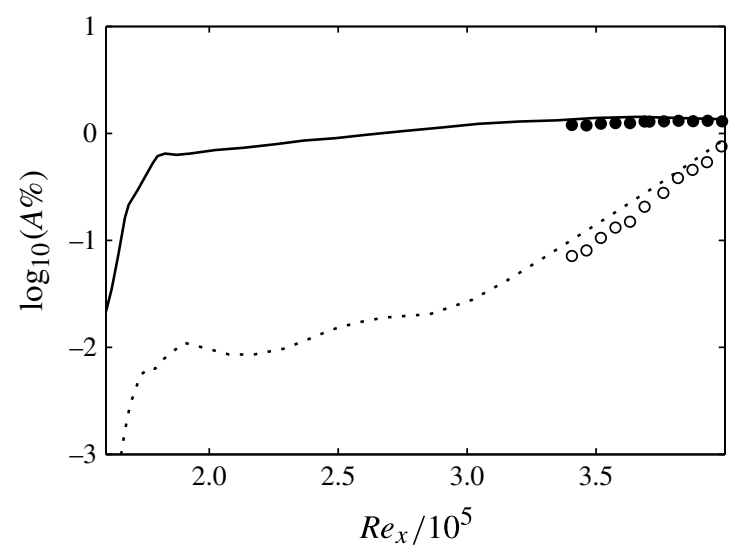

FIGURE 5. The percentage of the disturbance amplitude versus the downstream coordinate direction, represented by $R e_{x}: \bullet$, DMD mode with the frequency of the TS wave; O, DMD mode with subharmonic frequency; --, fundamental TS disturbance from DNS; $\cdots$, subharmonic disturbance from DNS.

linear and periodic signals, corresponding to global mode and Fourier decomposition, respectively (Chen, Tu \& Rowley 2012).

The two dominant low-frequency modes of the K-type transition are shown in figure 6. Since K-type transition occurs further upstream, a spatial extent of $2.05 \leqslant x / x_{0}=R e_{x} / 10^{5} \leqslant 2.35$ was chosen, to allow qualitative comparison with the slower $\mathrm{H}$-type transition. In contrast to the $\mathrm{H}$-type transition, analysed above, the three-dimensional wave now has the same frequency as the fundamental TS wave. While a DMD analysis of the H-type transition extracts the two distinct frequencies, no such distinction can be made for the K-type scenario. Rather, the existence of the three-dimensional wave is indirectly captured by a modulation of the two-dimensional fundamental wave with the superimposed three-dimensional wave. As before, we notice a marked increase in the three-dimensionality of both modes towards the right edge of the computational domain in figure 6, reflecting the spatial rise of the three-dimensional wave in the streamwise direction.

A final demonstration of the applicability of DMD to a transitional boundary layer involves the convergence of a superposition of dominant dynamic modes to the coherent vortical structures familiar from DNS. A subsection of the full domain for $\mathrm{K}$-type transition is chosen, where $\Lambda$-vortices are known to form. From our DNS, an example of this vortex is displayed in figure 7(a), visualized by the second invariant $Q$ of the velocity gradient tensor (Hunt, Wray \& Moin 1988). The reconstruction of this vortical structure using one, two and three low-frequency DMD modes is shown in figure $7(b-d)$ and illustrates good (quantitative) convergence towards the full $\Lambda$-vortex, further corroborating the ability of the DMD in capturing the important coherent structures.

Rempfer \& Fasel (1994) applied POD to the same transition scenarios (K- and H-type) and reported similar results. This is to be expected as, during the initial stages of transition, when the skin-friction coefficient is beginning to depart from its laminar correlation and $\Lambda$-vortices are starting to form, the coherent structures in the flow are energetically dominant. However, in the later stages, as smaller-scale structures begin to form, the difference between an energy-based POD and a frequency-based DMD 
(a)

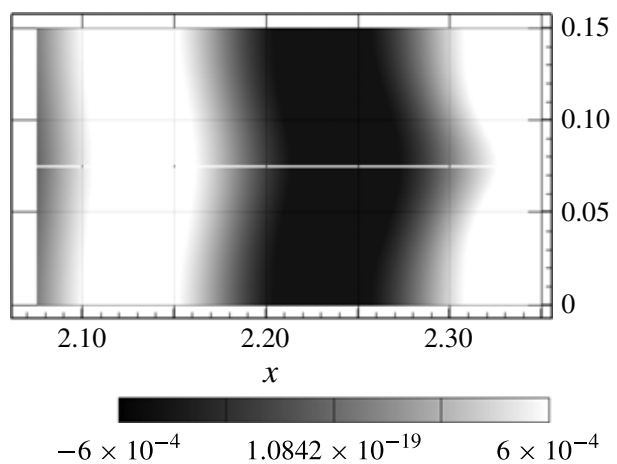

(b)

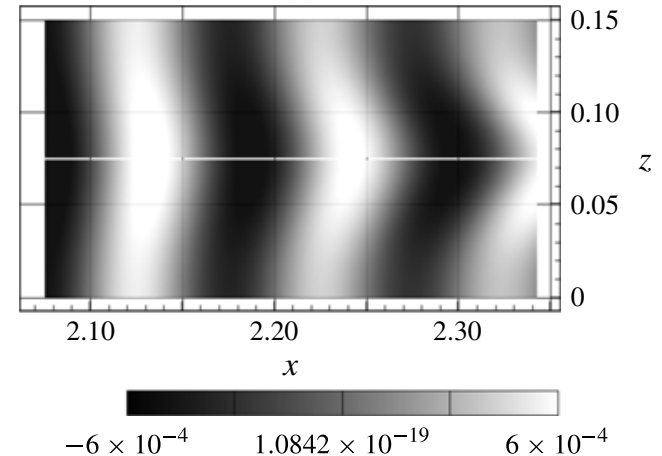

FIGURE 6. Contours of the wall-normal velocity at $y / x_{0}=0.01$ for the two dominant dynamic modes, extracted from the domain extending from $x / x_{0}=2.05$ to $x / x_{0}=2.35$ (K-type): (a) first low-frequency mode; $(b)$ second low-frequency mode.

(a)
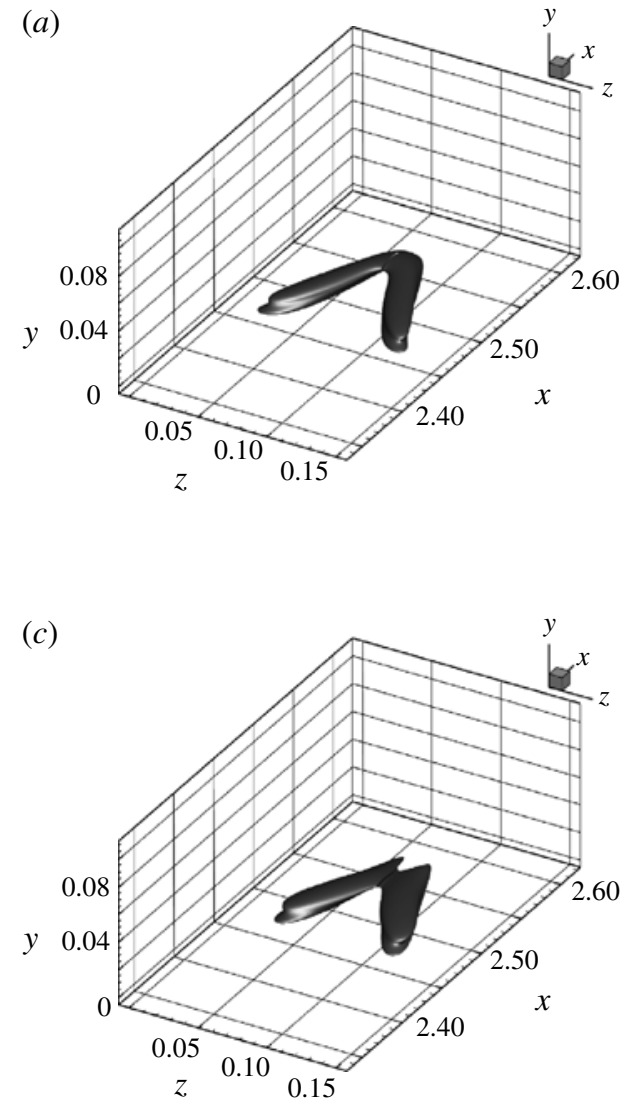
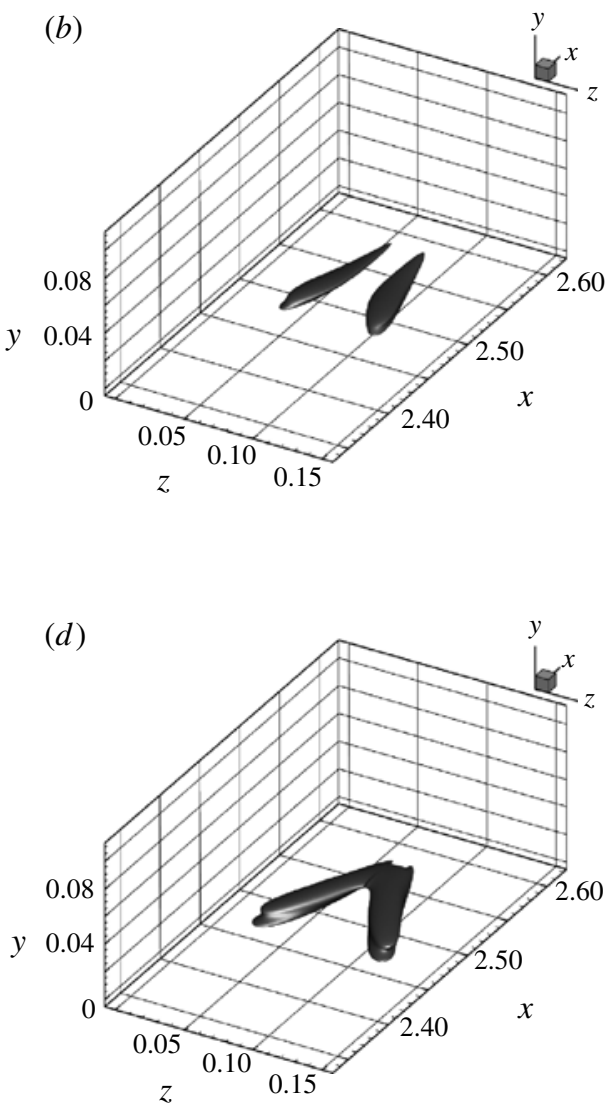

FIgURE 7. Isosurfaces of the $Q$-criterion showing a $\Lambda$-vortex (for K-type transition): (a) extracted from DNS, $(b-d)$ reconstructed by a superposition of one, two and three low-frequency dynamic modes. (a) DNS data. (b) One DMD mode. (c) Two DMD modes. (d) Three DMD modes. 
analysis increases. While the most energetic POD mode represents the entire vortex structure, a superposition of a few DMD modes is necessary to accomplish the full reconstruction of the $\Lambda$-vortex, which stems from the fact that different parts of the $\Lambda$-vortex travel at different convection velocities and are thus identified in different frequencies and as different dynamic modes. We will see below that this distinction of different parts of the $\Lambda$-vortices according to their convective speed will play an interesting role in their link to the associated skin friction at the wall and to its low-dimensional representation by low-frequency components.

\section{Structures extracted within the rise of the skin friction profile, DNS}

In this section triple decomposition is applied to the transitional flow in the spatial domain where the skin-friction rises and reaches a peak. Coupled with a DMD analysis, the triple decomposition allows an assessment of the contributions of each coherent structure (DMD mode) to the total Reynolds shear-stress profile. We will see that only a few low-frequency DMD modes, representing structures close to the wall, are needed to reconstruct the correct level of Reynolds shear-stress gradient through the transition. Furthermore, by employing composite DMD, a link between near-wall low-frequency structures and the skin-friction coefficient can be established.

\subsection{Triple decomposition: formalism}

Triple decomposition, proposed by Reynolds \& Hussain (1972), represents a flow field as a sum of three components according to

$$
f=\bar{f}+\tilde{f}+f^{\prime}
$$

where $\bar{f}$ is the time-averaged component, $\tilde{f}$ is the time-periodic wave and $f^{\prime}$ is the component attributed to broadband turbulence. Here, $\tilde{f}$ is defined by phase averaging the data with a specific frequency resulting in $\langle f\rangle$ such that $\tilde{f}=\langle f\rangle-\bar{f}$. Time and phase averaging of the incompressible Navier-Stokes equations results in

$$
\begin{gathered}
\frac{\partial \bar{u}_{i}}{\partial x_{i}}=\frac{\partial \tilde{u}_{i}}{\partial x_{i}}=\frac{\partial u_{i}^{\prime}}{\partial x_{i}}=0 \\
\bar{u}_{j} \frac{\partial \bar{u}_{i}}{\partial x_{j}}=-\frac{\partial \bar{p}}{\partial x_{i}}+\frac{1}{\operatorname{Re}} \frac{\partial^{2} \bar{u}_{i}}{\partial x_{j} \partial x_{j}}-\frac{\partial}{\partial x_{j}}\left(\overline{u_{i}^{\prime} u_{j}^{\prime}}\right)-\frac{\partial}{\partial x_{j}}\left(\overline{\tilde{u}_{i} \tilde{u}_{j}}\right) .
\end{gathered}
$$

The performance of a conventional time averaging operation on the momentum equation would have resulted in the same left-hand side and the first two terms on the right-hand side of (4.3). The third and fourth terms are combined into the Reynolds shear stress according to

$$
\frac{\partial}{\partial x_{j}}\left(\overline{u_{i}^{\prime t} u_{j}^{\prime t}}\right)=\frac{\partial}{\partial x_{j}}\left(\overline{u_{i}^{\prime} u_{j}^{\prime}}\right)+\frac{\partial}{\partial x_{j}}\left(\overline{\tilde{u}_{i} \tilde{u}_{j}}\right),
$$

where $u^{\prime t}$ is defined as $u^{\prime t}=u-\bar{u}, \partial / \partial x_{j}\left(\overline{u_{i}^{\prime} u_{j}^{\prime}}\right)$ is the contribution of the turbulent motion and $\partial / \partial x_{j}\left(\overline{\tilde{u}_{i} \tilde{u}_{j}}\right)$ denotes the contribution of the coherent motion to the Reynolds shear stress. Following the DMD procedure, each mode is characterized by a specific frequency. It can thus be argued that each dynamic mode represents a structure that can be thought of as phase-averaged over its respective modal frequency. 
While direct phase averaging requires many snapshots to combat spectral leakage arising from the sample frequency, DMD is able to extract the same information from a limited number of snapshots in a robust way. Dynamic mode decomposition allows the frequency to adjust, avoiding spectral leakage, and therefore is less sensitive to the particular choice of sample frequency and length. As a result, DMD is referred to as an optimal phase-averaging procedure. Based on this description, one can view the contribution of each dynamic mode to the Reynolds shear stress as the contribution of coherent motion from a specific phase-averaged structure. The contribution of the $n$th coherent structure to the total Reynolds shear stress is given by $\partial / \partial x_{j}\left(\overline{\tilde{u}_{i}^{n} \tilde{u}_{j}^{n}}\right)$, where $n$ represents the $n$th dynamic mode. The contribution of each mode can be separately added, as modes with different frequencies are decorrelated.

At this stage it is worth noting that the time signal associated with each POD mode is composed of multiple frequencies; as a result, in the context of triple decomposition it cannot be considered as a phase-averaged coherent structure, motivating the choice of DMD for this application. For a small number of snapshots, DMD outperforms the fast Fourier transform (FFT) as it does not suffer from spectral leakage and offers the flexibility of choosing a temporal range within which the snapshots are collected.

\subsection{Dynamic mode decomposition of the velocity field from DNS}

Based on velocity fields taken from the DNS calculations of $\mathrm{H}$ - and K-type transitions, we aim to investigate whether a few dynamic modes are able to reproduce the correct level of Reynolds shear-stress gradient through the entire transition process. In the vicinity of the wall the total stress is constant, and the Reynolds shear stress is directly related to the skin-friction coefficient. As a result, this study would have implications for the successful prediction of the skin-friction coefficient as well.

We choose a computational domain for H-type transition stretching from $x / x_{0}=5.5$ to $x / x_{0}=5.8$, covering the spatial region where the skin-friction coefficient departs from the laminar value and almost exhibits an overshoot to beyond the value of turbulent correlation (see figure 1). Similarly to the previous section, the three velocity components are analysed through DMD. The isosurfaces of the $Q$-criterion, additionally coloured by the streamwise velocity, are plotted in figure $8(e)$; packets of hairpin-shaped vortices are clearly visible within the domain. Figure $8(b-d)$ also displays contours of the wall-normal velocity at $y / x_{0}=0.01$. At this stage, the flow is nonlinearly saturated, causing all modes to lie close to the imaginary axis. This suggests that no significant growth or decay can be attributed to a mode. The modes are either of oscillatory nature if $\lambda_{i} \neq 0$ or are stationary if $\lambda_{i}=0$. As a result, the real part of the frequency is not plotted, and the DMD spectra are given based solely on the imaginary part of the frequency in figure $8(a)$. The three dominant dynamic modes (again, paired with their complex conjugate counterparts), as indicated in figure $8(a)$, show larger-scale structures consisting of several typical wavelengths in the spanwise direction. Despite the homogeneity in the spanwise $(z)$ direction, Fourier transform in $z$ would have resulted in non-compact spatial structures; this contrast highlights an advantage of a fully three-dimensional DMD.

The contribution of each identified mode to the total Reynolds shear stress can be readily computed by employing the triple-decomposition methodology outlined in section $\S 4.1$ and can be compared with the data from the DNS. In figure 9 we show, at a chosen streamwise location $x / x_{0}=5.7$, the contribution from a sum of the three dominant low-frequency modes and compare it with the total Reynolds shear stress of the DNS. The estimate of the total Reynolds stress from these three modes 

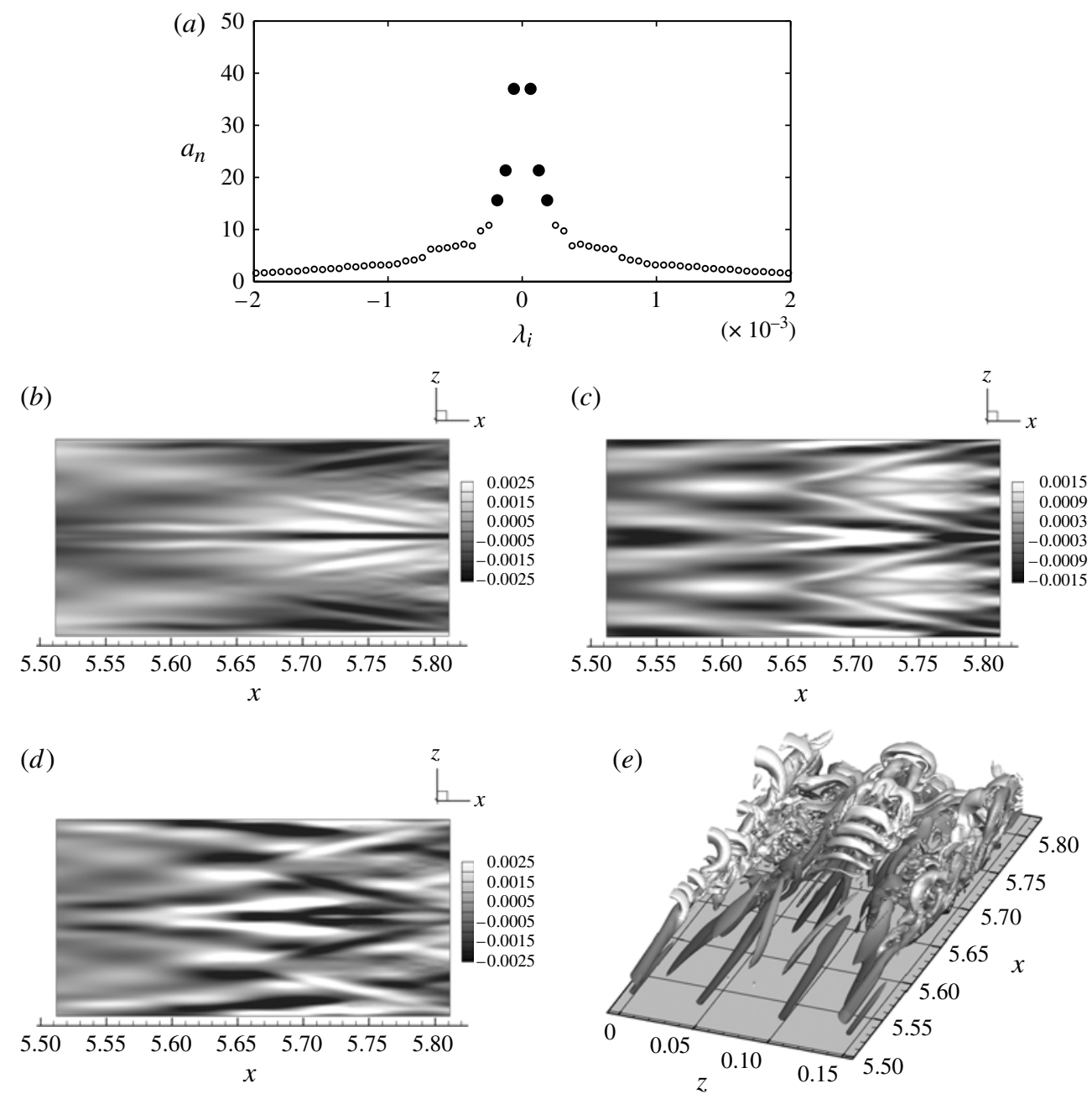

FIGURE 8. Dynamic mode decomposition of DNS velocity fields from H-type transition for $5.5 \leqslant x / x_{0} \leqslant 5.8$. (a) Amplitudes of the dynamic modes, $a_{n}$ : O, all DMD modes; -, three dominant low-frequency modes. $(b, c, d)$ Structures of the dominant low-frequency modes, visualized by contours of the wall-normal velocity at $y / x_{0}=0.01$. (e) Isosurface of the $Q$-criterion, from DNS coloured by the streamwise velocity.

yields a good approximation of the total value from DNS. It should be noted that the agreement is satisfactory throughout the transitional region and stays within $5 \%$ accuracy. These low-frequency modes represent large-scale flow structures, as observed in figure $8(b-d)$. To further investigate the flow structures contained in just these three modes, the flow field within the computational domain is reconstructed using the mean solution and these low-frequency modes; the results are shown in figure 10 and can be compared with results from DNS, shown in figure 8(e). The low-frequency modes contain streamwise elongated structures with an average spanwise spacing of $z^{+}=125$ in the wall-parallel plane at a distance of $y+=50$ from the wall. This is close to the typical spanwise spacing of near-wall streaky structures observed in fully developed turbulent boundary layer flow (Robinson 1991), although in the present transitional 


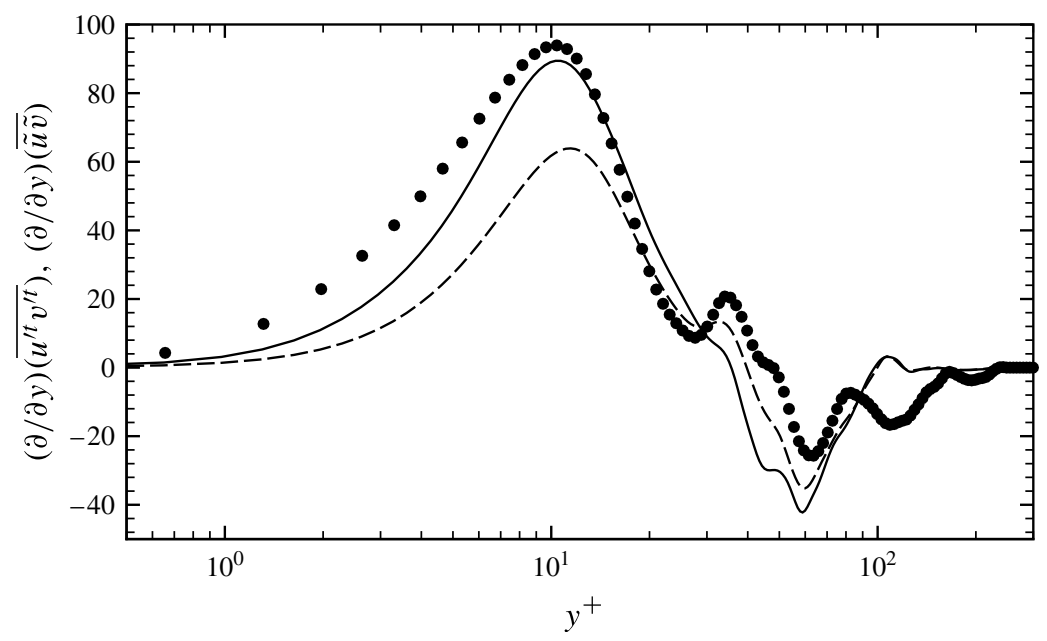

FIGURE 9. Shear stress profiles for H-type transition at $x / x_{0}=5.7$ : $\bullet$, total Reynolds shear stress from DNS; --, estimated Reynolds shear stress from a sum of the three dominant low-frequency modes; ---, estimated Reynolds shear stress from three filtered dynamic modes.

zone the structures are still harmonically influenced by the upstream forcing. We observe, however, that some of the small-scale elements and, more importantly, the tips of the hairpin vortices are not captured by the three-mode reconstruction. This is most evident from the vortex line plots in figure $10(a, b)$. The streamwise elongated vortical structures in these figures are nonetheless connected by a bridge as in typical hairpin-like structures, but this connection is of far lower strength than the heads of the hairpin vortices found in the high-frequency modes (present in the DNS). This finding agrees with the observations made in the near-wall region of turbulent wall-bounded flows (Jeong et al. 1997), where elongated streamwise vortices are dominant.

If these modes can be captured correctly on a coarse grid (e.g. in LES), the total shear stress can be estimated correctly as a consequence. Anticipating the application of the present decomposition to the data from LES, it is instructive to assess the influence of a coarse grid on these modes. Each dynamic mode is therefore filtered using a top-hat filter in the physical space with a filter width of $8 \Delta x$ and $8 \Delta z$, where $\Delta x$ and $\Delta z$ are the grid spacings along the streamwise and spanwise directions, respectively. This filter width coincides with the resolution of the grid, which is used in the UNS and LES calculations of table 1. In figure 9, the result is compared with the exact DNS shear stress as well as the shear stress estimated by the unfiltered low-frequency modes. Apparently, the DMD modes are affected by the filter. The difference between the filtered and the unfiltered shear stress profile has to be accounted for by an appropriate SGS model.

The same analysis can be performed for K-type transition. In this case, the subsection of the domain used to apply the triple decomposition extends from $x / x_{0}=3.4$ to $x / x_{0}=3.7$. As before, this domain coincides with the region close to the overshoot of the skin-friction profile and the onset of localized turbulence preceding fully turbulent fluid motion. The domain of interest is shown in figure 11(a). The isosurfaces of the $Q$-criterion, additionally coloured by the streamwise velocity 

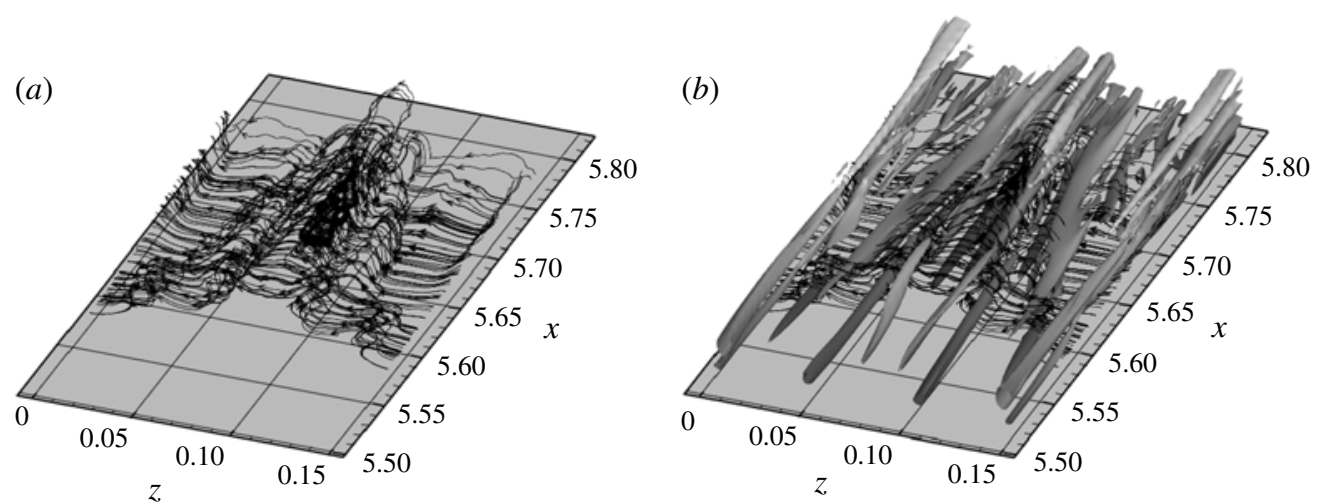

FIGURE 10. Vortex lines and isosurfaces of the $Q$-criterion, coloured by the streamwise velocity for H-type transition. (a) Vortex line representation of the flow field reconstructed from three dominant low-frequency dynamic modes. (b) The same as $(a)$, but visualized by the $Q$-criterion and the streamwise velocity component.
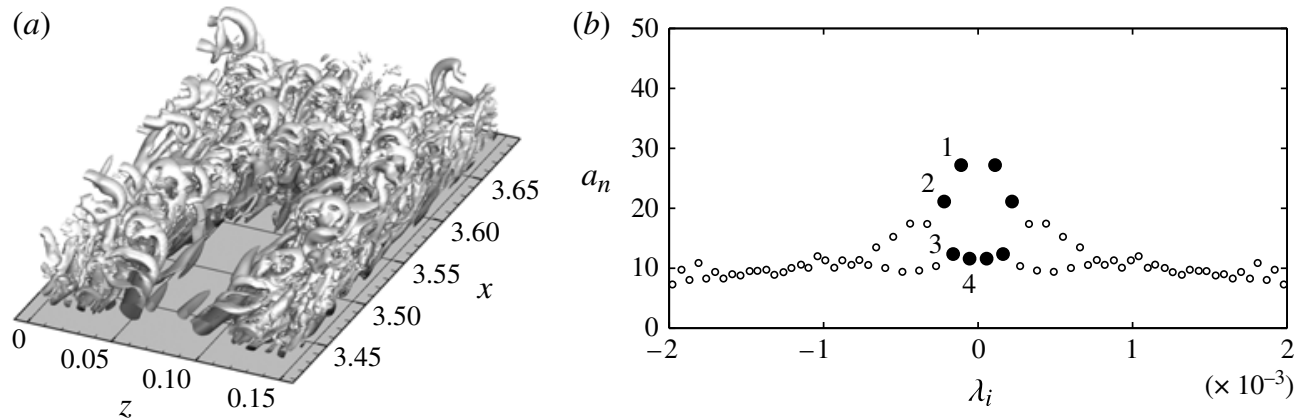

FIGURE 11. Numerical simulations capturing the rise of the skin-friction coefficient for K-type transition. (a) Isosurfaces of the $Q$-criterion and coloured streamwise velocity contours from DNS; $(b)$ amplitudes from the DMD, indicating four low-frequency modes by filled symbols.

component, illustrate the vortical structures present in the flow. The amplitudes of the first four low-frequency dynamic modes, determined from the K-type transition dataset, are shown in figure $11(b)$. The contributions of the four low-frequency dynamic modes are superimposed and compared with the total Reynolds shear stress in figure 12 at a streamwise location $x / x_{0}=3.6$. This superposition provides a good estimate of the shear stress, particularly close to the wall. Modes 3 and 4 have substantially lower amplitudes than modes 1 and 2; however, they provide a higher contribution to the total Reynolds shear-stress gradient. The contribution of each of these low-frequency modes to the total Reynolds shear-stress gradient is illustrated in figure 12. Sorting of the modes based on their amplitudes is not indicative of their contribution to the Reynolds shear-stress gradient as these amplitudes correspond to the signature of each mode on the velocity elements. 


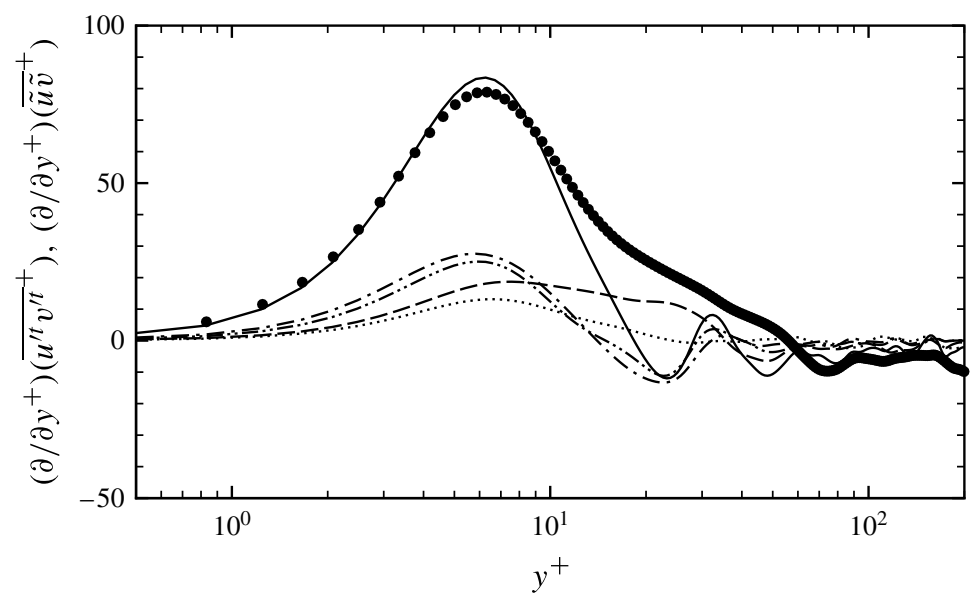

FIgURE 12. Shear stress profiles for K-type transition at $x / x_{0}=3.6$ : $\boldsymbol{\bullet}$, total Reynolds shear stress from DNS data; --, four low-frequency modes; ---, mode $1 ; \cdots$, mode 2; $-\cdot-$, mode $3 ;-\cdot \cdot-$, mode 4 .

In contrast to H-type transition, two of the dynamic modes for K-type transition, labeled 3 and 4, contain small-scale structures typical of a turbulent flow. Consequently, once the K-type modes are filtered using the same filter as above to illustrate the influence of coarsened resolution, the estimated shear stress from a superposition of four dynamic modes is drastically underpredicted. The contribution from SGS models for this transition scenario should therefore be larger than for the previous scenario, in agreement with the results of Sayadi \& Moin (2012), where it has been shown that even on the finer LES grid a higher value of turbulent eddy viscosity is reached for the K-type regime than for the H-type one.

In summary, only a few low-frequency dynamic modes are needed to provide a good estimate of the Reynolds shear stress gradient for both $\mathrm{H}$ - and K-type transitions. The prediction of the low-frequency modes remains within $5 \%$ of the total Reynolds shear stress in the region of interest, from the rise of the skin friction profile to the overshoot. This agreement deteriorates when the more turbulent region of the flow is considered. The predictive quality of these low-frequency modes deteriorates as the grid resolution decreases. This tendency has been illustrated by filtering the dynamic modes prior to reconstructing the Reynolds shear stress gradient. However, in this study the filtered modes have been obtained directly from the DNS calculation, where the influence of small-scale structures on the larger scales has been accounted for in the simulations. In a true LES this interaction is not captured in the same manner; a more relevant approach then would be to base the analysis and the mode extraction on lower-resolution calculations directly, which is the subject of $\S 5$.

\subsection{Dynamic mode decomposition of composite data, DNS}

The results of the previous section suggest that few low-frequency dynamic modes, computed from the DNS data, are capable of approximating the Reynolds shear-stress gradient. By visualizing the vortical components of the modes we concluded that the near-wall dynamics is governed by the legs of the $\Lambda$ - and hairpin vortices during the transitional regime. To quantitatively corroborate this observation, a DMD of composite data sequences is performed, where each data snapshot consists of 


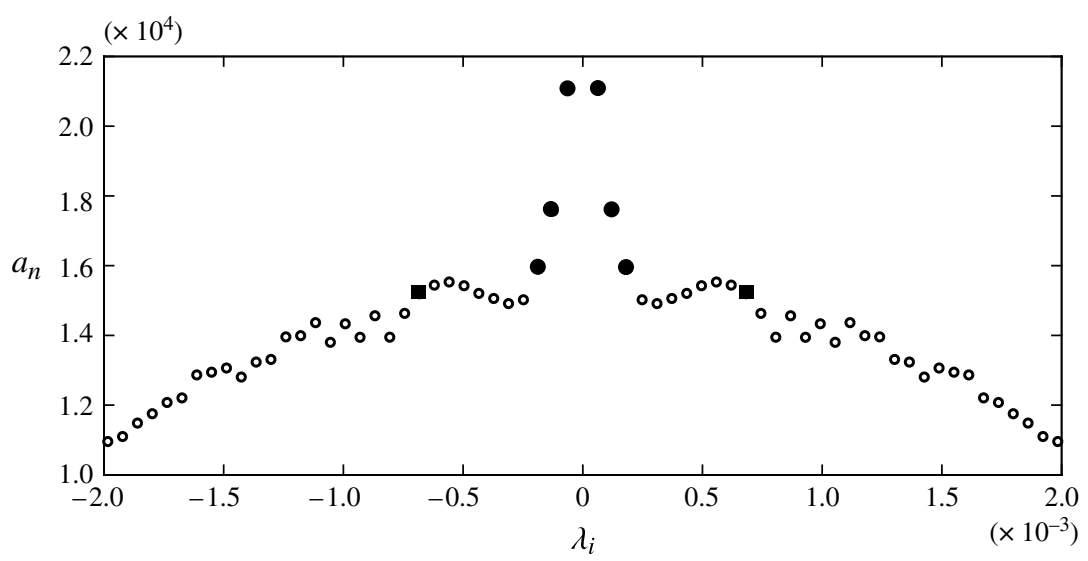

FIgURE 13. The amplitude distribution of the DMD of composite data for H-type transition: $\mathrm{O}$, all DMD modes; $\boldsymbol{0}$, three low-frequency modes; $\mathbf{\square}$, representative high-frequency mode.

the skin-friction coefficient on the wall and the field of the second invariant $Q$ of the velocity-gradient tensor. The $Q$-criterion is taken as a surrogate for vortical structures in the flow. The composite data are processed as before, and the dominant low-frequency composite modes are collected; the vortical part of the composite modes and their skin-friction footprint are then compared with the structures from the DNS and the total drag, respectively.

Figure 13 shows the amplitude distribution of the composite DMD performed for the H-type transition scenario. The computational subdomain for these calculations extends from $x / x_{0}=4.6$ to $x / x_{0}=5.8$. This region coincides with the location where $\Lambda$-vortices first appear in the flow and cause the skin friction to deviate from the laminar correlation to the region near the skin-friction overshoot. The subdomain studied previously in $\S 4$ is thus included in this domain. Figure 14(a) depicts the skin-friction coefficient from the DNS data. In figure 14(b) the reconstruction of the skin-friction distribution using the first three low-frequency dynamic modes (indicated by filled circles in figure 13) is shown. A comparison of these two figures verifies a close match of the large-scale structures of the skin-friction coefficient, as well as its total value. A DMD of composite $Q$-skin-friction data highlights the association of vortical structures with the observed skin-friction distribution. Figure 15(b) shows the vortical structures from the same composite modes that were able to faithfully reconstruct the skin-friction distribution in figure 14(b). The results from DNS data are juxtaposed in figure 15(a). The low-frequency modes reproduce the legs of the $\Lambda$ - and hairpin vortices in this part of the computational domain, in agreement with earlier observations made in $\S 4$. These structures represent large-scale fluid motion close to the wall and yield a good estimate of the skin-friction coefficient. The location of high skin friction coincides with the legs of the streamwise vortices in the flow, an observation also reported in Choi, Moin \& Kim (1993) and Kravchenko, Choi \& Moin (1993). As demonstrated in figure 10(a,b) through vortex lines, these elongated near-wall structures are connected further away from the wall through bridges resembling the tips of hairpin vortices. However, these bridges are of lower strength compared with the hairpin heads that appear in the DNS data. 


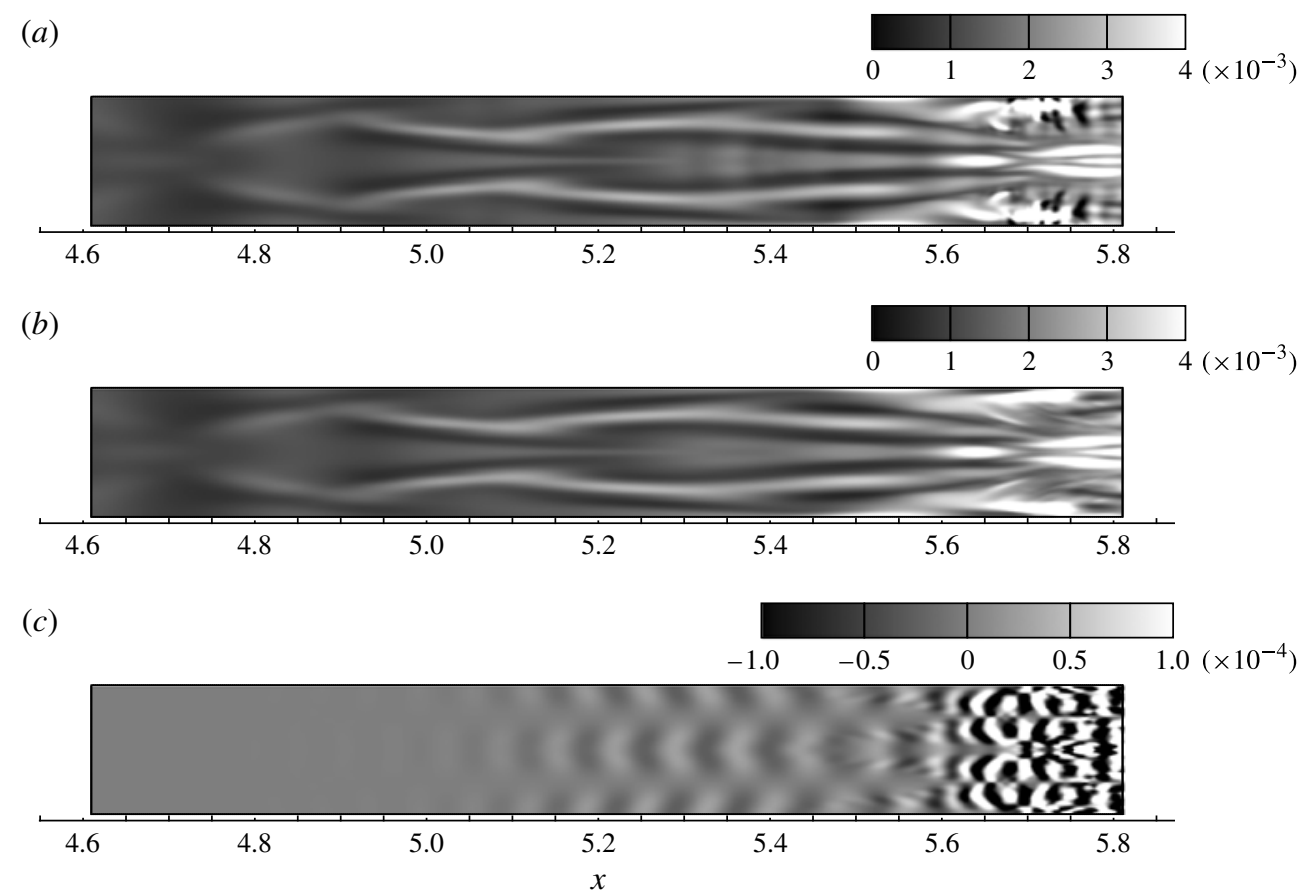

FIGURE 14. The skin-friction coefficient for H-type transition, comparing DNS $(a)$ with a reconstruction $(b)$ based on three low-frequency dynamic modes from composite data; (c) the skin-friction coefficient for a representative high-frequency dynamic mode.

In composite $\mathrm{DMD}$, the tips of the elongated near-wall structures appear with the correct strength in the high-frequency modes. A representative high-frequency mode, indicated by a filled square in figure 13, is displayed in figure 15(c). The tips are more numerous in the dynamic mode than in the DNS data, and their spacing is not exactly matched. This discrepancy can be accounted for by a superposition of a few high-frequency modes with nearly the same structure but different phases, such that the DNS data can be recovered instantaneously. The skin-friction signature of this mode is shown in figure 14(c). The contour levels for this plot have been chosen an order of magnitude smaller than for the DNS results. This analysis suggests that the tips of the hairpin-like vortices are correlated with small-scale structures further downstream and have a minor contribution to the near-wall dynamics. It is worth pointing out that the vortical structures of the low-frequency and high-frequency DMD modes displayed in figures $15(b)$ and $15(c)$ may appear similar, but in fact are associated with different values of the $Q$-criterion. This mismatch is also reflected in the different skin-friction contour levels shown in figures $14(b)$ and $14(c)$. If visualized with comparable contour levels, the high-frequency modes would show only a negligible contribution to the overall vortical structures. Nevertheless, far beyond the skin-friction overshoot we would anticipate that higher-frequency modes would rise up to comparable levels to those shown in figure 15(b) and establish a broadband spectrum characteristic of fully developed turbulence. In this regime a decomposition of the flow field into coherent single-frequency structures by DMD will lose its applicability and value. 

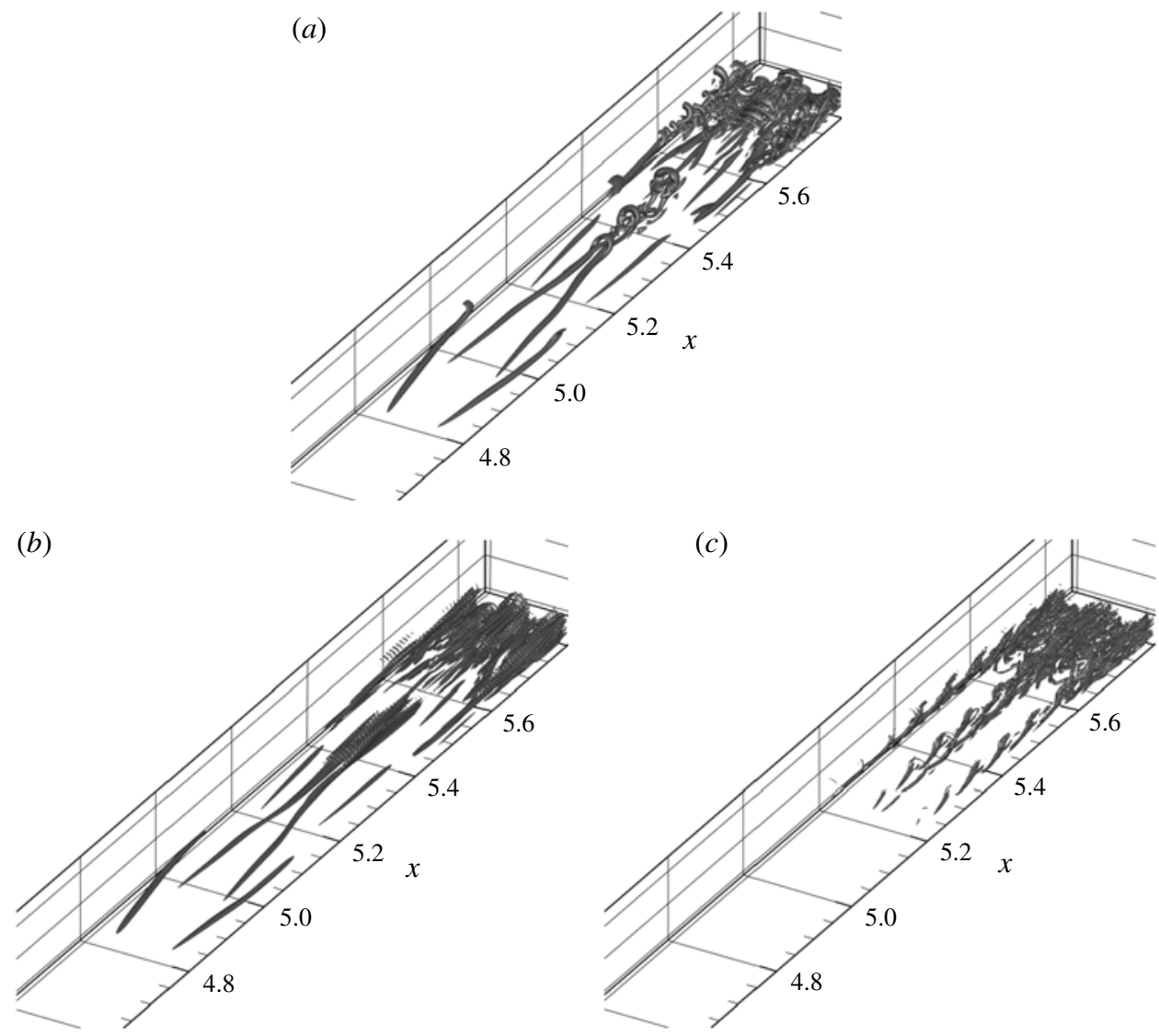

FIGURE 15. Isocontours of the $Q$-criterion for H-type transition, comparing DNS $(a)$ with a reconstruction $(b)$ based on three low-frequency dynamic modes from composite data; (c) vortical structures associated with a representative high-frequency dynamic mode.

\section{Structures extracted within the rise of the skin friction profile, LES and UNS}

Dynamic mode decomposition in the context of a triple decomposition is applied to the velocity elements of LES and UNS of H-type transition for the same flow configuration as the DNS described in the previous sections. The spatial mode shapes and their frequency in the transitional regime (coinciding with the rise of the skinfriction profile) are extracted and compared with the earlier results from the DNS. The LES and UNS underpredict the skin friction for this streamwise range, which can be explained by examining the contribution of the extracted low-frequency modes to the total Reynolds shear stress.

The DMD spectra for these three datasets are shown in figure 16(a). This figure shows that the frequencies of the modes are captured correctly through LES and UNS, as they match the DNS predictions. The structural contribution of these low-frequency modes to the total flow field is presented in figure 16(b). These structures should be compared with those of the DNS in figure 15(b), where similar features have been identified, specifically in the near-wall region. As expected, due to the limited resolution of LES and UNS, small-scale structures are absent in the reconstructed data. This was anticipated from our analysis of the filtered modes in $\S 4.2$. 


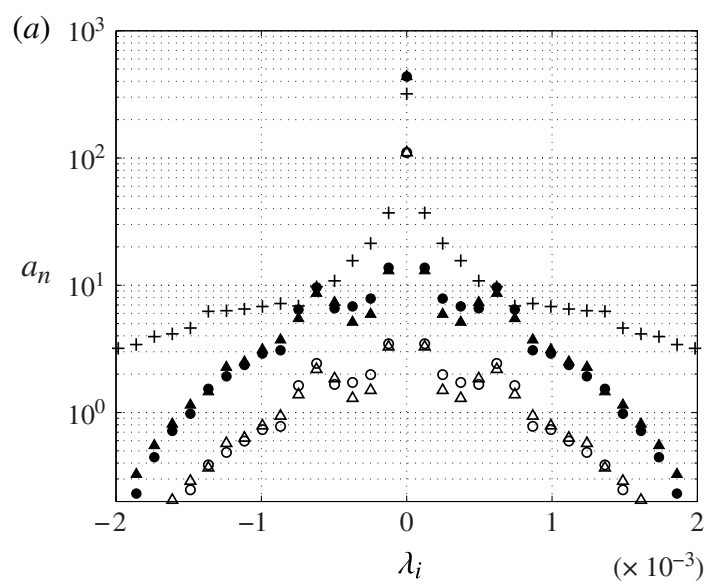

(b)

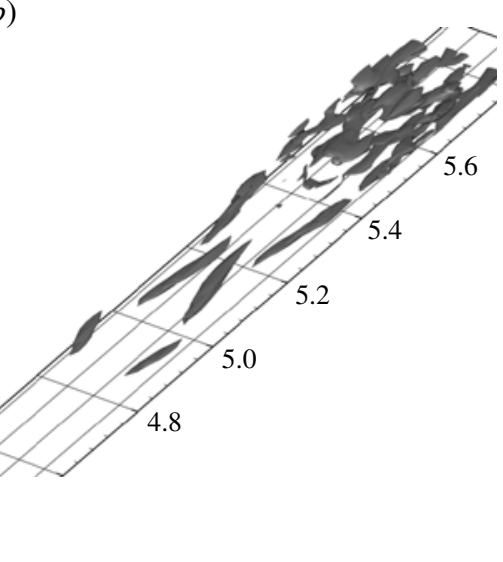

FIGURE 16. Dynamic mode decomposition applied to the low-resolution LES. (a) DMD spectra: O, LES; $\Delta$, UNS; +, DNS; ○, scaled LES; $\boldsymbol{\Delta}$, scaled UNS. (b) Isosurfaces of the $Q$-criteria, for the first three low-frequency modes.

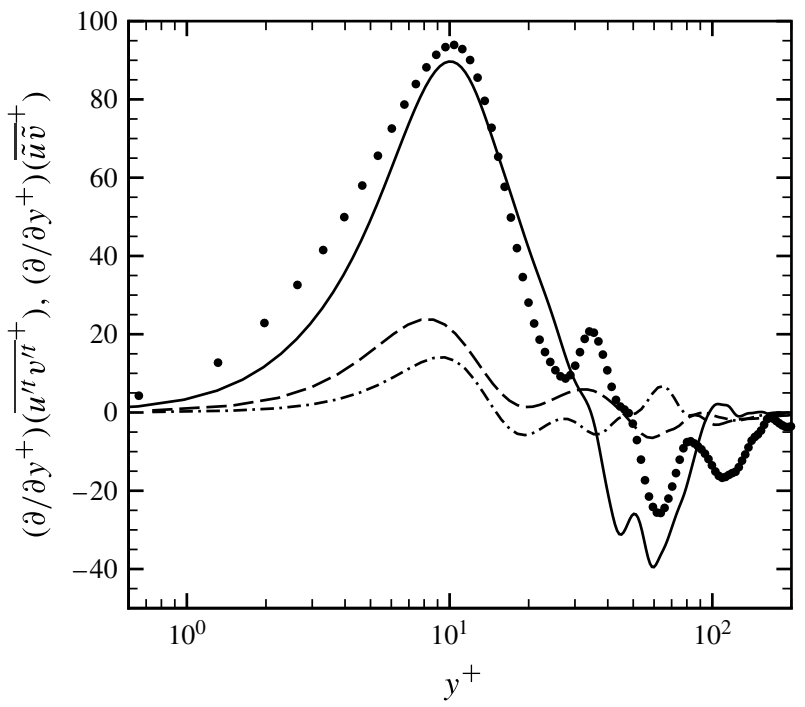

FIGURE 17. The Reynolds shear stress gradient: - DNS; --, low-frequency DMD modes from DNS; ---, low-frequency DMD modes from LES; -- -, low-frequency DMD modes from UNS.

In the previous section, we demonstrated that low-frequency modes give a good estimate of the Reynolds shear-stress gradient in this domain. Therefore, the first three low-frequency DMD modes are selected to construct the Reynolds shear-stress gradient at $x / x_{0}=5.7$, as shown in figure 17. The three modes from LES and UNS underpredict this value dramatically when compared with DNS. This is due to the underprediction of the magnitude of each DMD mode, as can be noticed from figure $16(a)$. 
(a)

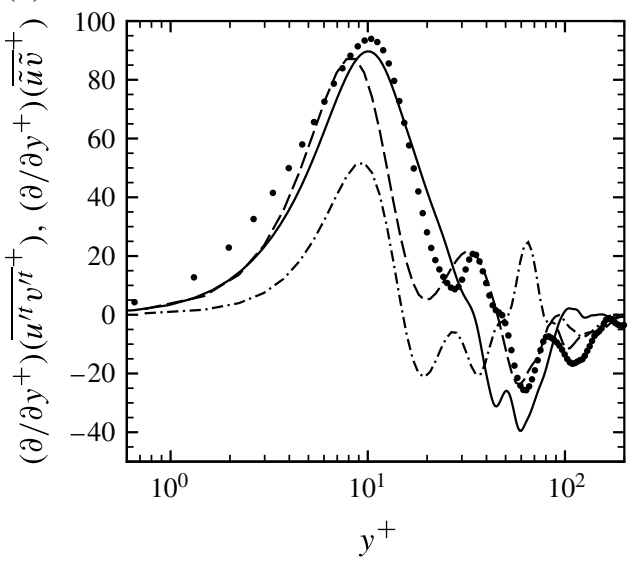

(b)

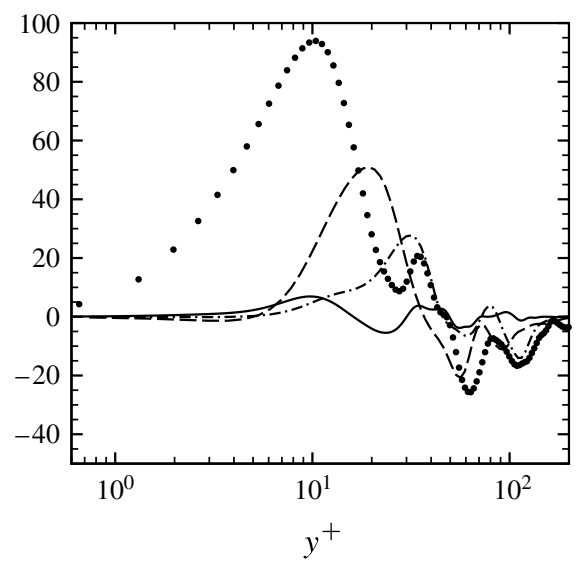

FIGURE 18. The Reynolds shear stress gradient: $\bullet$, DNS; --, DMD modes from DNS; --- , DMD modes from LES; -- -, DMD modes from UNS. (a) Rescaled low-frequency modes. (b) High-frequency mode.

In order to determine whether the underprediction of the Reynolds shear stress is due to the underpredicted magnitude and not the modal shape itself, we first define a coefficient of approximated energy based on the integral of the DMD spectra, defined as

$$
E_{D M D}=\int a_{n}\left(\lambda_{i}\right) \mathrm{d} \lambda_{i}
$$

The scaling coefficient is then defined as the ratio of the approximated energy of the DNS to that of the LES. All LES amplitudes are then scaled based on this ratio. The scaled spectrum is shown in figure 16(a) by the black symbols. This figure shows that the prediction of the amplitude of the mean is improved noticeably. The contribution to the total Reynolds shear stress can be scaled in a similar way, and the results are shown in figure 18(a). This figure demonstrates that while rescaled LES modes reasonably approximate the total Reynolds shear-stress profile, the performance of the UNS is less satisfactory and the Reynolds shear-stress profile remains underpredicted. The fact that the LES modes recover the shape of the DNS wall-normal Reynolds shear stress to within a rescaling factor is encouraging from a modelling perspective.

Figure 16 shows a kink in the spectra of the LES and UNS which is not apparent in the DNS spectra. The contribution to the total Reynolds shear stress from this relatively high-frequency mode is shown in figure $18(b)$. At this frequency, the DNS mode has a very small contribution to the total Reynolds shear-stress value. The mode from UNS has a higher contribution while the LES mode contributes the most to the Reynolds shear stress at this frequency. This suggests that through the LES model higher-frequency modes are artificially magnified, apparently at the expense of dynamically more important lower-frequency modes.

\section{Summary and conclusions}

Dynamic mode decomposition has been applied to DNS data for the controlled $\mathrm{H}$ - and K-type transitions to turbulence in a compressible boundary layer. This type of flow field decomposition yields coherent structures with a single (complex) 
frequency and has been able to accurately describe the flow fields in the early transitional regime by a reduced-order representation. The spatial growth or decay of structures in the downstream direction of the early transitional regime could be readily captured. A triple decomposition of the flow fields in conjunction with the DMD has given insight into the mechanisms and physical processes responsible for the divergence of the skin-friction coefficient from its laminar value towards a peak value. A low-dimensional model containing only a few low-frequency dynamic modes has been demonstrated to reproduce the relevant flow dynamics and to predict the correct levels of Reynolds shear-stress gradients, irrespective of the type of transition scenario (H- or K-type). A decomposition of composite data containing both vortical and skin-friction information revealed that only the legs of the hairpin-like vortices that occurred contributed significantly to the large-scale skin-friction distribution. The heads of the same vortices consisted of higher frequencies and could be linked to small-scale fluctuations of the skin-friction coefficient further downstream.

Large-eddy simulations commonly underpredict the level of Reynolds shear stresses during the transition process. The effect of a diminished resolution on the dynamically important structures has been assessed by applying this methodology to LES and UNS of the H-type transition. The analysis of LES data showed that the shape and frequencies of these low-frequency modes are predicted independently of the resolution. However, the underprediction of the amplitudes of these modes leads to an underprediction of the Reynolds shear-stress gradient. The DMD spectra illustrate a kink in the high-frequency spectrum of lower-resolution LES and UNS. Analysis of the contribution of this higher-frequency mode suggests that through the LES model higher-frequency modes are artificially magnified at the expense of dynamically more important lower-frequency modes.

\section{Acknowledgements}

Computational support was provided by the Argonne Leadership Computing Facility, the PSAAP center supported by DOE NNSA and the LaSIPS project.

\section{REFERENCES}

Aubry, N., Holmes, P., Lumley, J. L. \& Stone, E. 1988 The dynamics of coherent structure in the wall region of a turbulent boundary layer. J. Fluid Mech. 192, 115-173.

BaKe, S., Fernholz, H. H. \& Kachanov, Y. S. 2000 Resemblance of K- and N-regimes of boundary-layer transition at late stages. Eur. J. Mech. (B/Fluids) 19 (1), 1-22.

BAKEWELL, H. P. JR. \& LUMLEY, J. L. 1967 Viscous sublayer and adjacent wall region in turbulent pipe flow. Phys. Fluids 10 (9), 1880-1889.

Berlin, S., Wiegel, M. \& Henningson, D. S. 1999 Numerical and experimental investigations of oblique boundary layer transition. J. Fluid Mech. 393, 23-57.

Borodulin, V. \& KaCHANOV, Y. 1995 Formation and development of coherent structures in a transitional boundary layer. J. Appl. Mech. Tech. Phys. 36 (4), 532-564.

Borodulin, V. I., Kachanov, Y. S. \& RoscheKtayeV, A. P. 2011 Experimental detection of deterministic turbulence. J. Turbul. N23.

Chen, K. K., TU, J. H. \& Rowley, C. W. 2012 Variants of dynamic mode decomposition: boundary condition, Koopman, and Fourier analyses. J. Nonlinear Sci. 22 (6), 887-915.

Choi, H., Moin, P. \& KIM, J. 1993 Direct numerical simulation of turbulent flow over riblets. J. Fluid Mech. 255, 503-539.

Head, M. R. \& BANDYOPADhyay, P. 1981 New aspects of turbulent boundary-layer structure. J. Fluid Mech. 107, 297-338. 
Holmes, P., Lumley, J. L. \& Berkooz, G. 1996 Turbulence, Coherent Structures, Dynamical Systems and Symmetry, Cambridge Monographs on Mechanics. Cambridge University Press and McGraw-Hill.

Hunt, J. C. R., Wray, A. A. \& Moin, P. 1988 Eddies, stream, and convergence zones in turbulent flows. Center for Turbulence Research Report. CTR-S88, pp. 193-208.

Hussain, A. K. M. F. 1986 Coherent structures and turbulence. J. Fluid Mech. 173, 303-356.

JeOng, J., Hussain, F., SCHOPPA, W. \& KIM, J. 1997 Coherent structures near the wall in a turbulent channel flow. J. Fluid Mech. 332, 185-214.

Kachanov, Y. S. \& Levchenko, V. Y. 1984 The resonant interaction of disturbances at laminarturbulent transition in a boundary layer. J. Fluid Mech. 138, 209-247.

Klebanoff, P. S., Tidstrom, K. D. \& Sargent, L. M. 1962 The three-dimensional nature of boundary-layer instability. J. Fluid Mech. 12, 1-34.

Kline, S. J., Reynolds, W. C., Schraub, F. A. \& Runstadler, P. W. 1967 The structure of turbulent boundary layers. J. Fluid Mech. 30, 741-773.

Kravchenko, A. G., Choi, H. \& Moin, P. 1993 On the relation of near-wall streamwise vortices to wall skin friction in turbulent boundary layers. Phys. Fluids A 5 (12), 3307-3309.

MezIC, I. 2005 Spectral properties of dynamical systems, model reduction and decompositions. Nonlinear Dyn. 41 (1), 309-325.

Mizuno, Y., Duke, D., AtKinson, C. \& Soria, J. 2011 Investigation of wall-bounded turbulent flow using dynamic mode decomposition. 13th European Turbulence Conference, vol. 318, p. 042040 .

Moin, P. \& Moser, R. D. 1989 Characteristic-eddy decomposition of turbulence in a channel. J. Fluid Mech. 200, 471-509.

Nagarajan, S., Lele, S. K. \& Ferziger, J. H. 2003 A robust high-order compact method for large eddy simulation. J. Comput. Phys. 191 (2), 392-419.

REMPFER, D. \& FASEL, H. F. 1994 Evolution of three-dimensional coherent structures in a flat-plate boundary layer. J. Fluid Mech. 260, 351-375.

Reynolds, W. C. \& Hussain, A. K. M. F. 1972 The mechanics of an organized wave in turbulent shear flow. Part 3. Theoretical models and comparisons with experiments. J. Fluid Mech. 54 (02), 263-288.

RIST, U. \& FASEL, H. 1995 Direct numerical simulation of controlled transition in a flat-plate boundary layer. J. Fluid Mech. 298, 211-248.

Robinson, S. K. 1991 Coherent motion in the turbulent boundary layer. Annu. Rev. Fluid Mech. 23, 601-639.

Rowley, C. W., Mezic, I., Bagheri, S., Schlatter, P. \& Henningson, D. S. 2009 Spectral analysis of nonlinear flows. J. Fluid Mech. 641, 115-127.

SARIC, W. S. 1986 Visualization of different transition mechanisms. In Gallery of Fluid Motion (ed. H. L. Reed), Phys. Fluids 29, 2770.

SAYADI, T. 2012 Numerical simulation of controlled transition to developed turbulence in a zeropressure-gradient flat-plate boundary layer. $\mathrm{PhD}$ thesis, Stanford University.

Sayadi, T., Hamman, C. W. \& Moin, P. 2013 Direct simulation of complete H-type and K-type transitions with implications for the structure of turbulent boundary layers. J. Fluid Mech. 724, 480-509.

SAYADI, T. \& MoIN, P. 2012 Large eddy simulation of controlled transition to turbulence. Phys. Fluids 24 (11), 114103-114117.

Sayadi, T., Nichols, J. W., Schmid, P. J. \& Jovanovic, M. R. 2012 Dynamic mode decomposition of H-type transition to turbulence. In Center for Turbulence Research, Proceedings of the Summer Program, pp. 5-14.

SCHMID, P. J. 2010 Dynamic mode decomposition of numerical and experimental data. J. Fluid Mech. 656, 5-28.

Schmid, P. J., Violato, D. \& Scarano, F. 2012 Decomposition of time-resolved tomographic PIV. Exp. Fluids 52, 1567-1579. 
Schubauer, G. B. \& SKramstad, H. K. 1947 Laminar boundary layer oscillations and stability of laminar flow. J. Aeronaut. Sci. 14, 69-78.

Sirovich, L. 1987 Turbulence and the dynamics of coherent structures. Quart. Appl. Math. 45, 561-590.

Spalart, P. R. \& WatmufF, J. H. 1993 Experimental and numerical study of a turbulent boundary layer with pressure gradients. J. Fluid Mech. 249, 337-371.

White, F. M. 2006 Viscous Fluid Flow. 3rd edn. McGraw-Hill International Edition.

WU, X. \& MoIN, P. 2009 Direct numerical simulation of turbulence in a nominally zero-pressuregradient flat-plate boundary layer. J. Fluid Mech. 630, 5-41. 